\title{
Building Learning Repository based on Semantic and Shareable Learning Objects
}

\author{
Khaled M. Fouad \\ Computer Science Dept., \\ College of Computers and \\ Information Technology, Taif \\ University, Kingdom of Saudi \\ Arabia (KSA)
}

\author{
Mostafa A. Nofal \\ Computer Engineering Dept., \\ College of Computers and \\ Information Technology, Taif \\ University, Kingdom of Saudi \\ Arabia (KSA)
}

\author{
Jehan A. Hasan \\ Computer science Dept., \\ College of Education and \\ Science, Taif University, \\ Kingdom of Saudi Arabia
}

(KSA)

\begin{abstract}
Learning Objects (LO) represent important elements when using electronic media to deliver educational contents to an audience in a learning environment.Construction of LOs does not involve a simple method, as their nature is directly linked to the intricacies of human cognitive and learning processes.Learning Objects Repository (LOR) not only provides a distributed storage mechanism but also emphasizes on the shareability and reusability of LOs. To ensure reusability and shareability, the metadata has been annotated to help LO discovery. To enhance the annotations in a traditional metadata format, ontological approach is proposed to achieve efficient LOs and to assist users in retrieving LOs. In this paper, new approach is proposed to acquiresemantic shareable learning objectsto create LOR. This proposed system presents the advantages that facilitate the development of new didactic tools with improved LO retrieving, integration and building capabilities.The experiment executed in this research shows that the proposed approach improves LO retrieving in the learning system.
\end{abstract}

\section{General Terms}

E-Learning, Learning objects (LO), Shareable learning objects,Learning object metadata (LOM), Domain ontology, WordNet.

\section{Keywords}

Learning object repository, Semantic Web, Domain ontology, Semantic learning objects, Semantic annotation, LO refinement.

\section{INTRODUCTION}

When an E-Learning system to be delivered contains learning materials covering different levels of learning, the level of learner is taken into consideration to provide the learner with the learning materials that suit his/her level and his/her fields of interest [1]. In advanced e-learning systems, shareable learning objects [47] will be presented as a way for sharing information in standard ways that will allow the design of learning events that fit the needs of learners and provide justin-time opportunities to develop skills and knowledge. Learning Object Repositories [48] (LORs) are an important element in the management, publishing, location and retrieval of LOs.

Using ontology [2, 3] in learning environments aims at providing mechanisms to enhance the process of searching and finding learning resources. And, they have the capability to organize and display information that makes it easier for learners to draw connections, for instance, by visualizing relationships among concepts and ideas.The annotations [10] mean comments, notes, explanations, or other types of external remarks that can be attached to any Annotations are very helpful if they are inserted into documents containing many terms specific to subject. Semantic annotation [25] provides a more precise description of the knowledge contained in a document and it's semantics in the domain. Semantic annotation $[25,26]$ is about assigning entities in the text links to their semantic descriptions. The present research first proposes anapproach that extends the IEEE LOM (Learning Object Metadata) standard [9] with ontology-based semantic annotations [23] for efficient use of learning objects (LOs) outside learning management systems (LMS). This approach is presented in the context of existing approaches that adopt ontologies in order to annotate e-learning resources. Its particularity consists in a simple solution for integrating semantic annotations in the structure of the IEEE LOM [9] description of the e-learning resources. The proposed approach aims at building learning repository based on semantic and shareable LO. The approach provides new steps towards the unification of existing learning object conceptualizations. Based on the new formal semantic web specifications, proposed approach provides new room for interchanging, reusingand sharing of web-oriented educational contents in the form of learning objects. These advantages facilitate the development of new didactic tools with improved searching, integration and building capabilities.

\section{RELATED WORK}

Semantic Annotation Platforms (SAPs) [11] were developed to provide a level of automation to the semantic labeling process, and overcome the limitations of manual annotation. SAPs designed with extensible architectures can adapt to evolving technology. Information extraction components can be replaced as different approaches are developed. The most common toolkits used within SAPs are GATE and Amilcare.

In [12], authors have reviewed the existing principled methods for semantic annotation. Specifically, authors focused on two most important issues in semantic annotation: entity extraction and relation extraction. For entity extraction, they introduce four state-of-the-art methods: rule based methods, classification based methods, sequential labeling based methods, and non-linear based Markov random fields based methods. For relation extraction, they also introduce four typical methods: classification based methods, kernel based method, sequential labeling based methods, and other methods. They have explained the principle of these methods 
by using several approaches as examples. Authors have described several annotation systems and compared the main features and the algorithms employed in the systems. Moreover, we have introduced several practical application of semantic annotation.

Authors in [13] proposed to utilize data mining technologies for time series data to gather the relevant information, such as citations, of specific LOs in different timescales. That is, citations in different timescales represent different meaning of the LOs. They revised the Time-Fading Model and Tilt-Time Window Model to measure the weight of LOs. In addition, authors provided a mechanism to rank these LOs. Utilizing the proposed mechanism, it can enhance reusability of LOs. Furthermore, to assist users in the searching phrase, they revised the algorithm of Relevance Feedback and combined it with the weight of LOs that we proposed. They did not provide actual items, like the recommendation systems, to users. Instead, they provided suggestions that can guide them to revise the query process especially in the input query terms. They proved that, with the proposed mechanisms and the distance learning standard that focuses on describing LOs (i.e., IEEE LOM); LOs can be searched in an efficient way, which will help the promotion of SCORM and CORDRA specifications in the international community of distance learning.

The authors in [14] applied the graph-theoretic clustering to Web data for noising, clustering and constructing semantic concept model. They proposed a novel image annotation method. The proposed method offline processes and mines Web data collected from several image search engine to construct a concept clustering model. Moreover, it can be continuously upgraded; the effectiveness of image annotation can be increased gradually with the development of the update phase. They demonstrated that it can obviously improve the effectiveness of annotation algorithm through offline deleting the noise data and mining the important terms in Internet data.

The aims in [15] were to create a system that annotates automatically a set of Web pages according to domain ontology. They were interested in data-rich web pages which are pages that contain a list of records. Most of these pages are automatically generated conforming to a template. This property makes possible extracting data they contain using wrapper induction techniques. They tried to associate it with different sets of data units' concepts or properties that best match it. Their approach was based, among other things, on syntactic descriptions of concepts and properties with which they have enriched the ontology.

In the paper [16], authors presented an approach for the automatic generation of metadata for LOs. Main ingredients of their model were the ontology and the classifier. The classifier automatically classifies the LOs according to the concepts in the given ontology. Classified LOs were then automatically attached with ontological metadata. The proposed approach is easy to implement and reduces the amount of manual intervention while integrating the LOs with metadata.

In the paper [17], authors presented a system to semantically annotate any named entity contained in a text, using a URI link. The URI resource used is a standard one, compatible with the Semantic Web network Linked Data. They have introduced the concept of Linked Data Interface, an exhaustive statistical resource containing contextual and nature description of potential semantic objects to label. The Linked Data Interface gives a possible answer to solve the problem of ambiguity resolution for an exhaustive semantic annotation process. This system is a functional proposition, available now, to establish automatically a relation between the vast amount of entry points available on the Linked Data network and named entities contained in an open text.

The specific purposes of the study [18] were to (1) implement an online social annotation tool, HyLighter, for questionanswering tasks in a college argument and persuasion course, and (2) investigate the effects of HyLighter on the students' mental models, motivation for the course, and achievement in the course, compared to the students who engaged in question-answering tasks without using HyLighter.

Authors in [19] have proposed an automated video annotation method which is able to detect and label the foreground region of interest using appearance and motion information. More specifically, they focused on annotating rigid moving objects such as cars, airplanes, etc. in Web videos, and they considered videos with only one foreground object present. Authors proposed to construct consensus foreground object templates (CFOT) to address moving object detection. Their method was robust to significant camera motions (e.g. panning, tilting, zooming, etc.) or low contrast environments. They also considered the integration of features collected in different domains, which further improves the annotation accuracy.

In [20], authors discussed some of the most important problems that occur when creating semantic annotations of multimedia/textual resources during the CIPHER project. First, relevant formalisms, like frames, semantic networks and description logics, were introduced. The discussion in this chapter showed the most advantageous choice for creating semantic annotations. In spite of the difficulties of description logics to model relations, they provided a well-defined semantics that opened the door to advanced and useful services, like modeling error explanations. The subsequent sections in the chapter presented the state of the art in error explanation techniques for OWL Web Ontology Language and the possibility of solving the problems with modeling relations in OWL.

\section{BASIC CONCEPTS}

\subsection{E-Learning and Semantic Web}

Electronic learning [27] is interactive learning in which the learning content is available online and provides automatic feedback to the student's learning activities.

It is generally agreed that credible evidence for mastery of learned material [28] is the goal of instruction. While educators and domain experts agree that decoding meaning from text plays a critical role in the acquisition of knowledge across all disciplines, what particular evidence of mastery is required and what lends credibility to such evidence are the subjects of a lively debate among experts in the learning community. The need for new methods for semantic analysis of digital text is now widely recognized in the face of the rising tide of information on the Web.

The layered model [29] for the Semantic Web, as found in Figure 1, puts the relationship among ontology description languages, RDF and RDF Schema, and XML in a better perspective. The bottom layer offers character encoding (Unicode) and referencing (URI) mechanisms. The second layer introduces XML as the document exchange standard. The third layer accommodates RDF and RDF Schema as mechanisms to describe the resources available on the Web. 
As such, they may be classified as lightweight ontology languages. Full ontology description languages appear in the fourth layer as a way to capture more semantics. The topmost layer introduces expressive rule languages. The brief history of ontology description languages in this section expands the above comments. It was introduced the concept of a markup language in 1967. Very briefly, a markup language indicates the structure of a document with help of tags intermixed with the data.

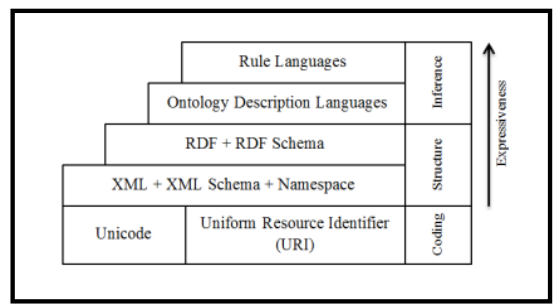

Fig 1: Architecture for the Semantic Web.

The semantic web [21] is a space understandable and navigable by both human and software agents. It adds structured meaning and organization to the navigational data of the current web, based on formalized ontologies and controlled vocabularies with semantic links to each other. From the E-Learning perspective, it aids learners in locating, accessing, querying, processing, and assessing learning resources across a distributed heterogeneous network; it also aids instructors in creating, locating, using, reusing, sharing and exchanging learning objects (data and components). The semantic web-based educational systems need to interoperate, collaborate and exchange content or re-use functionality. A key to enabling the interoperability is to capitalize on (1) semantic conceptualization and ontologies, (2) common standardized communication syntax, and (3) large-scale service-based integration of educational content and functionality provision and usage. The vision of the semantic web-based E-Learning is founded on the following major premises (1) Machine-understandable educational content, (2) Shareable educational ontologies, (3) Educational semantic web services, and (4) Semantic interoperability.

Interoperability requires the use of standard SW languages for representing ontologies, educational content, and services.

\subsection{Learning Object (LO) and Learning Object Repository (LOR)}

The Learning Object (LO) [30] is derived from object oriented programming conception. The object oriented emphasizes on the reusability of objects. According to the idea that LO can share itself to other instructors, the instructor can also reuse them on different teaching materials. Based on the framework, it is not hard to image that a new updated LO which has been stored in a specific system in network, could be immediate used by others. This kind of Learning Object Repository LOR system can greatly reduce the developmental cost, accelerate the development of teaching materials, and easy to download or integrated other application by other users conveniently. The major characteristic of learning object transformed by a standardized process can be recomposed to a new learning resource. While a teaching material stored in LOR, it implements standardized structure means to divide the teaching material into small learning unit and stored in individual location of LOR. It is one of the most differences between traditional teaching materials.
It was provided a classification of types of learning objects, making it easier to understand the nature of the elements contained inside of them [5]:

- Fundamental - The basic, most simple form of learning aid. A simple image depicting a stage of a surgical procedure

- Combined-closed - Still a simple element but one that integrates more complex mechanisms in order to provide an explanation. An animation or video clip depicting a surgicalprocedure, including audio

- Combined-open - Several simple objects encased inside integration elements. An integration element (i.e. a website) that includes the image and the video clip of the surgicalprocedure,along with the use of plain text that explains the procedure

- Generative-presentation - Combination of objects providing advanced visual and auditive capabilities with limited interactive features. A dynamic Flash animation capable ofgenerating and recreating a visual picture depicting a surgical procedure and its inherent operational conditions

- Generative-instructional - Combination of objects providing advanced visual and auditive capabilities with advanced interactive features, allowing a high level of hands-on experience.

A dynamic Flash animation, linked to an image and text database, capable of generating a graphic environment depicting a surgical procedure and its operational environment, where users can manipulate surgical instruments and monitor patient vital signs in order to provide hands-on instruction.

Still, it was previously noted that learning objects are dynamic in terms of their nature. Technology is not static and its inherent evolving characteristics suggest several aspects regarding the properties of learning objects that need to be taken into account. Table 1 shows those properties in detail as found in [6].

Table 1: Goals of Learning Objects [6]

\begin{tabular}{|l|l|}
\hline Goal & Description \\
\hline Reusability & $\begin{array}{l}\text { Learning content modularized into small units } \\
\text { of instruction suitable for assembly and } \\
\text { reassembly into a variety of courses }\end{array}$ \\
\hline
\end{tabular}

Interoperability Instructional units that interoperate with each other regardless of developer or learning management system

Durability Units of instruction that withstand ever evolving delivery and presentation technologies without becoming unusable

Accessibility Learning content that is available anywhere, anytime learning content that can be discovered and reused across networks

The rules of developing Learning Object have been emerged in [22]. First of the rules is based on assumption that Learning Object is an autonomous didactical unit. Second guideline refers to use of educational standards and pedagogical theories upon designing and using Learning Object. Examples those standards are SCORM, IEEE LOM and the group of the AICC standards. Next concept is related to the problem of 
preserving a coherent context across a group of Learning Objects. When we connect Learning Objects we also associate specific context with them. It is possible to realize a situation where all the interconnected Learning Objects will be considered as mutually inconsistent, in other words taken of out their context.

\subsection{Learning Objects Metadata (LOM)}

Several metadata [7, 31] standards have emerged for the description of learning resources. The Dublin Core metadata initiative (DCMI, http://dublincore.org/) is an open forum engaged in the development of interoperable online metadata standards that supports a broad range of purposes and business models. Although Dublin Core attributes that contains metadata such as authors, title or granularity, are definitely useful for describing educational resource content, but Dublin Core does not contain attributes describing the pedagogical perspective of a document. In order to cope with the educational concerns, various metadata standards were defined such as IMS Metadata (IMS, http://www.imsglobal.org/), SCORM Metadata (SCORM, http://www.adlnet.gov/scorm/index.cfm), CanCore (http://www.cancore.ca/) and IEEE Learning Object Metadata (IEEE LOM,http://ltsc.ieee.org/wg12/index.html).

\section{THE PROPOSED SYSTEM}

To increase the reusability and the quality description of a learning object, it is necessary to use more specialized metadata than those proposed by the LOM specifications.Metadata can also be defined as descriptive and classifying information about the object. Learning object metadata (LOM) standard specifies a conceptual model that defines the structure of a metadata instance for a learning object.In order to enable effective reusing and repurposing of LOs, we have to further enhance semantics of their content. Here, an approach is recommendedto build upon the traditional LO creational schema, but further extends it to incorporate support for semantic structuring and markup of LO content using content structure and domain ontologies, respectively.

In our approach, LOs' metadata are presented in accordance with metadata ontology (MO). This metadata can be enriched with the concepts from the domain ontologies (DO). Each LO is assigned an ontology-based description of its structure [23], whereas its content is enriched with references to the concepts of one or more domain ontologies (DO).Semantic annotation approach also is proposed [24]. WorldNet used as ontology to acquire the relation between different keywords. Figure 2 shows the proposed system architecture.

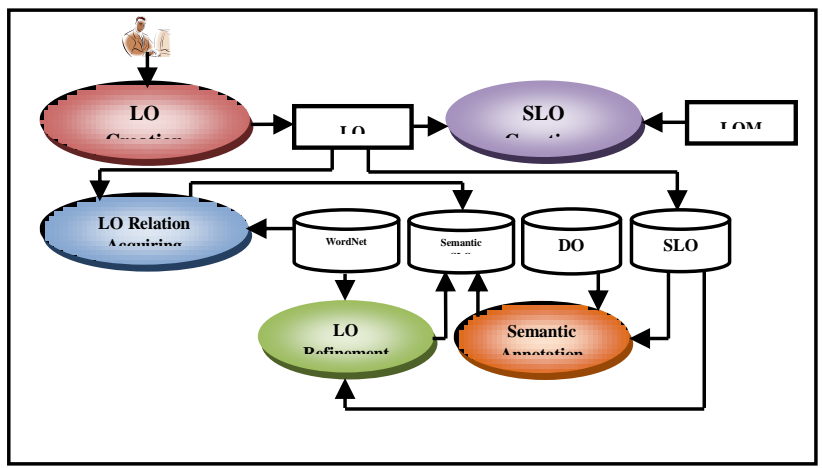

Fig 2: The proposed system architecture
The present research first proposes a model that extends the IEEE LOM (Learning Object Metadata) standard with ontology-based semantic annotations for efficient use of Learning Objects (LOs) outside Learning Management Systems (LMS). This model is presented in the context of existing approaches that adopt ontologies in order to annotate e-learning resources. Its particularity consists in a simple solution for integrating semantic annotations in the structure of the IEEE LOM description of the e-learning resources.

\subsection{LO Creation}

As mentioned in [32], authors have determined that the reusable learning objects is a reusable chunk of content with the following two fundamental properties: first is instructionally sound content with focused learning objectives. Second property is the facility that allows the learner to practice, learn, and receive assessment. Also they define the sharable learning objects SLOas reusable learning objectsRLO with the additional interoperability property that is the metadata or keywords that describe the object's attributes and mechanisms for communicating with any elearning system.

The aim of learning objects [35] creation is selecting and extract as much of the existing raw content into RLO. The methodology is an iterative five step process to select appropriate content for the RLO with opportunities to refine and re-structure as the extraction is taking place. The steps are shown in figure 3 .

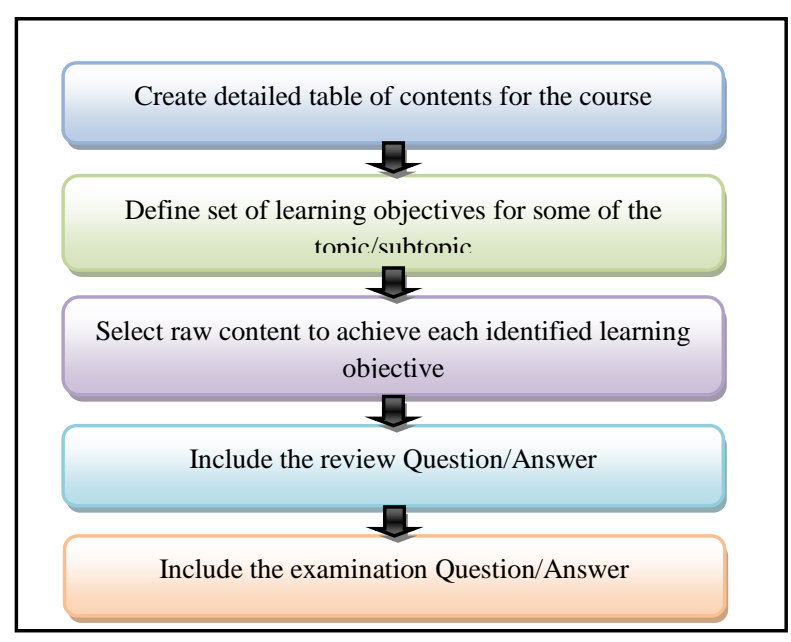

Fig 3: Steps for creating RLO

\subsection{SLO Creation}

In order to create sharable learning objects(SLO) [40] from RLO, the metadata must be added. The metadata will describe its properties which can be used to determine how should interact with LO. The metadata is also used to facilitate discovery of the SLO when it is stored in digital repository. IMS Learner Information Package Specification corresponding to some IEEE LOM [9] standard has been chosen. The IEEE LOM specification consists of nine categories, which includes 60 data elements. Although the lack of clarity in the IEEE LOM standard makes its value spaces hard to interpret, most metadata editors today continue to use that standard without seeking to explain the meaning of each space [33]. This issue is addressed to enable suitable learning objects management data to be introduced into learning environments by devising a set of definitions to clarify the content of each value space in the LOM.To design 
the metadata schema, the IEEE LOM standard has been followed [9]. A subset of metadata have been identified from the IEEE LOM specification, which are relevant for finding the suitability of a document to a particular e-learner and can automatically be extracted from learning materials [34, 36]. Recent work [9] provides standardization of LO metadata: the LOM framework is already part of the Sharable Content Object Reference Model [37] and all this effort, despite of some criticism, is expected to speed up the creation of learning objects repositories, that is large pools containing retrievable LO and metadata indexes based on the standard [38].

\subsection{LO Annotation}

In general, annotation [10] is a process of document enrichment with additional information especially in form of notes or comments. This information has to be created or collected, stored and then inserted back in some form into the document to be presented to the user. Semantic Annotation [39] of textual document is to identify the concept with the help of domain ontologies. For this purpose, a robust text analysis technique is required which will be composed of identification of object in a text, identification of relationship betweenthese objects and analysis on how these objects and their relationships combine toform a concept. Hence the objective of Semantic Annotation is to tag ontology class instances foundin the text using text analysis process and map it into ontology classes as depictedin.

In [41], authors presented a survey of current semantic annotation platforms that can be used to perform semiautomatic annotation. The presented platforms vary in their architecture, information extraction tools and methods, initial ontology, amount of manual work required to perform annotation, performance and other features, such as storage management.Figure 4 shows the algorithm of the semantic annotation in the proposed system.

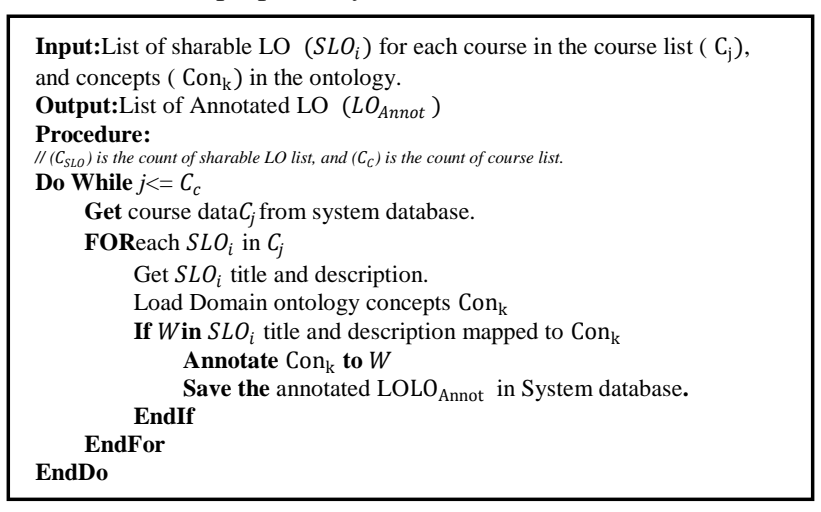

Fig 4: The algorithm of the semantic annotation

\subsection{LO Refinement}

LO refinement aims at determining the redundancy found in the generated LO by analyzing LO titles using semantic similarity. In the proposed system, the LO refinement is performed by extracting the most similar LO from the generatedLO by calculating the semantic similarity for each LO. Semantic similarity $[42,49]$ used in the proposed system is based on WordNet [43] to refine the generated LO. The used method considers the LO titles as sentence and get the semantic similarity between them to refine generated LO. Figure 5 shows the algorithm of LO refinement.

\subsection{LO Relation Acquiring}

In WordNet [43], Synsets are interlinked by means of conceptual-semantic and lexical relations. Various semantic relationships such as synonym, antonym, hyperonym, meronym are represented among the synsets. In order to utilize the WordNet for learning taxonomical (hyperonym) relations, one must solve the problem of identifying the word sense, by using word sense disambiguation WSD [44], so that the synonym, hyperonym, hyponym of the word in LO in that sense could be located in the hierarchical synsets.

In the proposed system, this method aims at extracting high quality domain-specific concepts using WordNet. With this concept extraction as the basis, WordNet based method is presented for taxonomy extraction using WordNet [43] and word sense disambiguation [44].

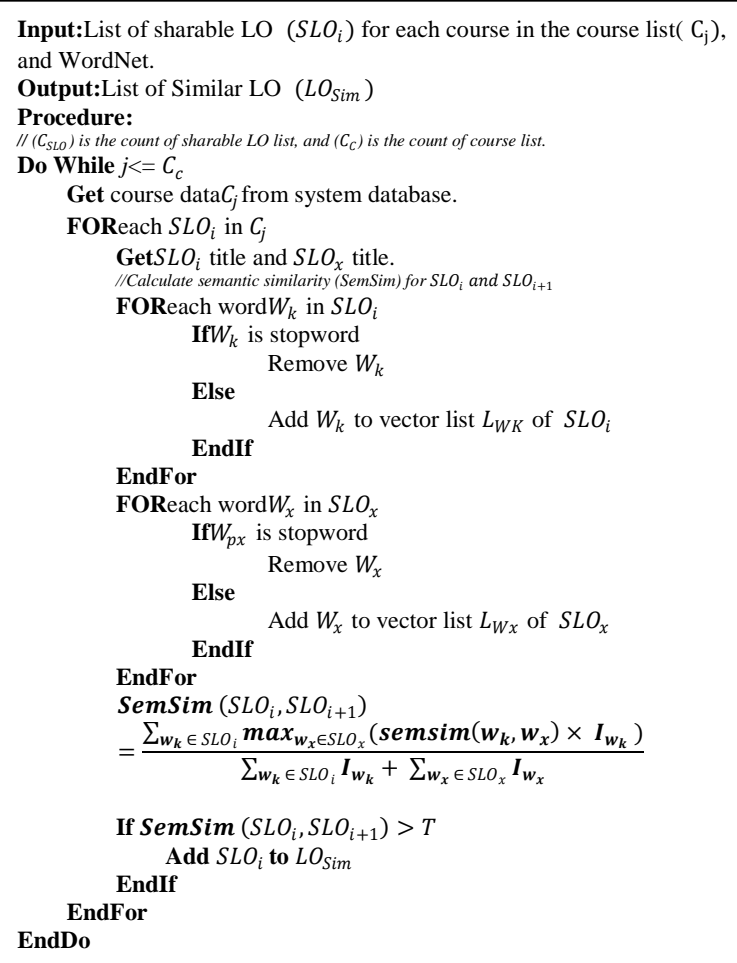

Fig5:The algorithm of $\mathrm{LO}$ refinement

\subsection{The Domain Ontology}

The main reason for ontology [45] is to enable communication between computer systems in a way that is independent of the individual system technologies, information architectures and application domain. It is used mainly to index the relevant learning objects and to facilitate semantic search and re-usability of learning objects.

It was proposed in [46] a knowledge engineering approach to build domain ontology. Figure 6 shows main steps of the ontology development process.

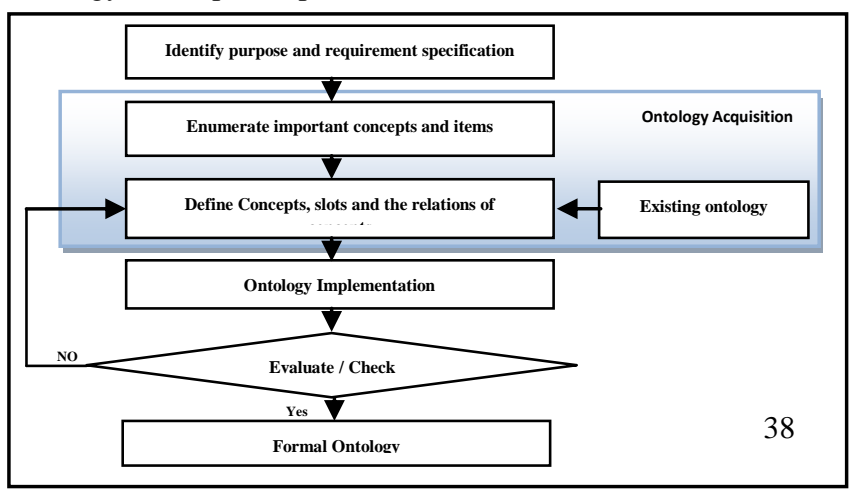




\subsection{System Database}

The system database contains the required data such as courses and sharable learning objects. Figure 7 shows the database diagram that is implemented in SQL Server 2008.

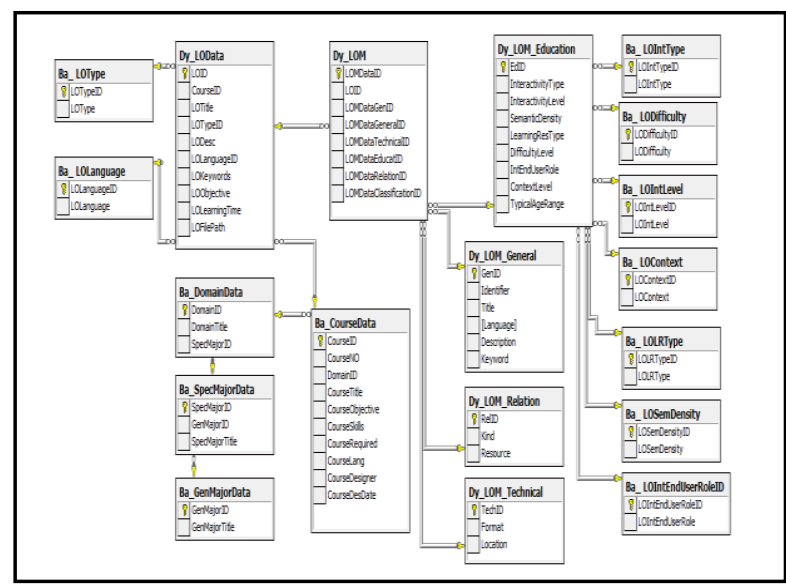

Fig 7: The system database diagram

\section{SYSTEM EVALUATION}

In this section, the results of the experiments carried out to evaluate the performance of proposed system is discussed from a quantitative point of view by running some experiments to evaluate the precision of the results.

The proposed system is implemented in ASP.Net as Webbased system using Visual Studio 2010, .NET Framework 4, and SQL Server 2008. The number of stored courses is 3 courses that have276 LOs. These courses are about computer science domain. Figure 8 shows the samples of the system

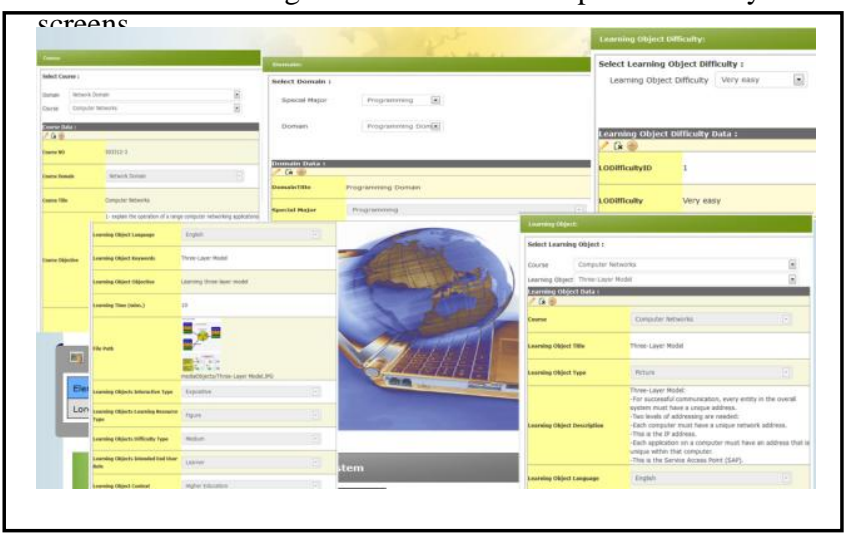

Fig8:Samples of the system screens

Figure 9 shows the ontology editor of the selected domain.

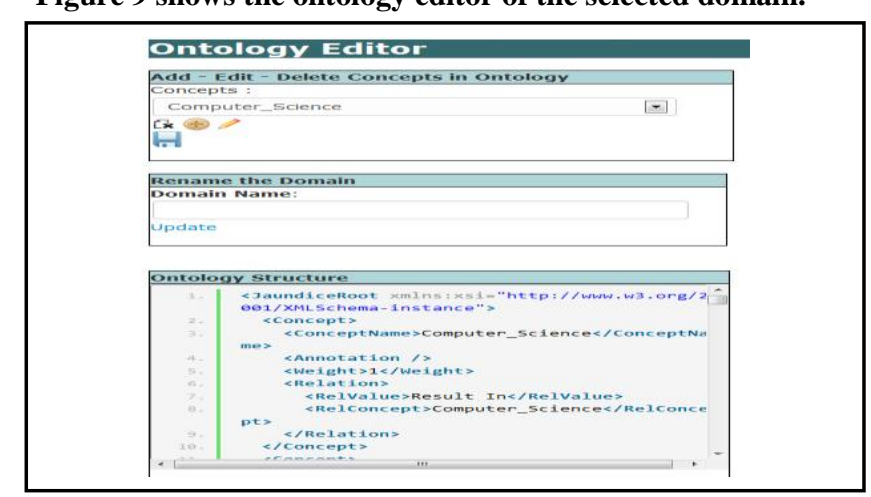

Fig9:The ontology editor

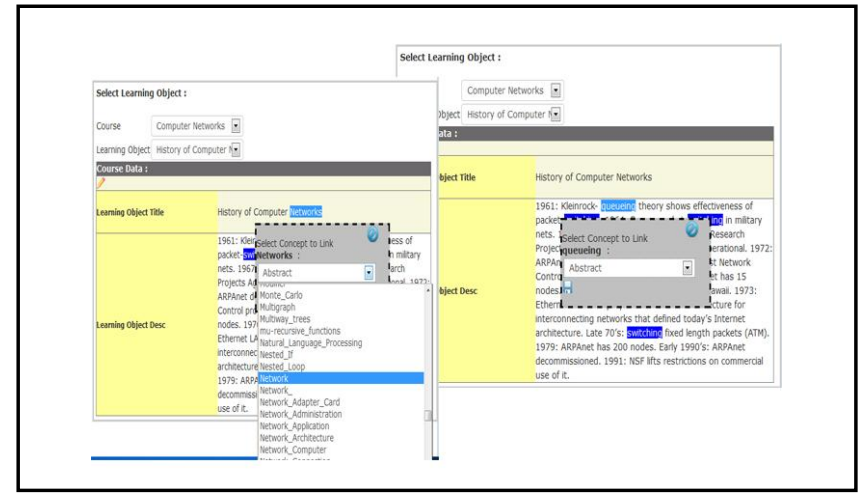

Fig10:LO annotation

In order to evaluate the efficiency of the proposed method, the computer science domain ontologyhas been constructed by ontology editor that is created in the system. Based on semantic web, a prototype system has been designed and implemented. In this research, recall ratioP $P_{\text {recall }}$ and precision ratio $\mathrm{P}_{\text {precision }}$ are applied to evaluate efficiency and accuracy of retrieving results. $P_{\text {recall }}$ refers to proportion of retrieved related $\mathrm{LO}\left(\mathrm{LO}_{\mathrm{RR}}\right)$ out of all related $\mathrm{LO}\left(\mathrm{LO}_{\text {AllRel }}\right)$ in the system. $\mathrm{P}_{\text {precision }}$ is defined as proportion of retrieved related $\mathrm{LO}\left(\mathrm{LO}_{\mathrm{RR}}\right)$ relative to all retrieved $\mathrm{LO}\left(\boldsymbol{L} \boldsymbol{O}_{\text {AllRet }}\right)$.

$$
\mathrm{P}_{\text {recall }}=\frac{L \boldsymbol{O}_{R R}}{L \boldsymbol{O}_{\text {AllRel }}}
$$

$$
\mathrm{P}_{\text {precision }}=\frac{L O_{R R}}{L O_{\text {AllRet }}}
$$

In order to analyze the efficiency, the comparison among the syntax based LO creating, semantic based LO creating is accomplished. Table 6 shows the experiment that appears the efficiency of the semantic based method for creating the learning objectsrepository in learning system.

Table 3 shows the samples of the experiment data and the calculated $\mathrm{P}_{\text {recall }}$ and $\mathrm{P}_{\text {precision }}$ for keyword based method and table 4 shows the data for semantic based method.

Table 3: The data of keyword based method

\begin{tabular}{lllll} 
LO $_{\text {AllRet }}$ & $\mathrm{LO}_{\text {AllRel }}$ & $\mathrm{LO}_{\mathrm{RR}}$ & $\boldsymbol{P}_{\text {recall }}$ & $P_{\text {precision }}$ \\
\hline 10 & 9 & 3 & 0.3333333 & 0.3 \\
20 & 18 & 9 & 0.5 & 0.45 \\
50 & 47 & 32 & 0.6808511 & 0.64 \\
70 & 67 & 52 & 0.7761194 & 0.74286 \\
100 & 88 & 61 & 0.6931818 & 0.61 \\
120 & 105 & 76 & 0.7238095 & 0.63333 \\
150 & 140 & 82 & 0.5857143 & 0.54667 \\
\end{tabular}

Table 4: The data of semantic based method

\begin{tabular}{lll|ll}
$L O_{\text {AllRet }}$ & LO $_{\text {AllRel }}$ & $L O_{R R}$ & $P_{\text {recall }}$ & $P_{\text {precision }}$ \\
\hline 10 & 9 & 7 & 0.778 & 0.7 \\
20 & 18 & 14 & 0.778 & 0.7
\end{tabular}




\begin{tabular}{lllll}
50 & 47 & 40 & 0.851 & 0.8 \\
70 & 67 & 60 & 0.896 & 0.857143 \\
100 & 88 & 85 & 0.966 & 0.85 \\
120 & 105 & 98 & 0.933 & 0.816667 \\
150 & 140 & 127 & 0.907 & 0.846667 \\
\hline
\end{tabular}

Figure 11 shows the difference of recall for each keyword based methodand semantic based method.

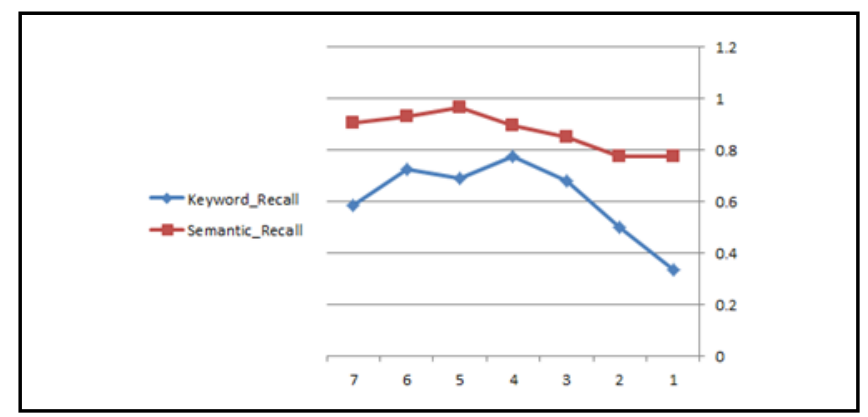

Fig11:Recall difference between keyword and semantic

Figure 12 shows the difference of precision for each keyword based method and semantic based method.

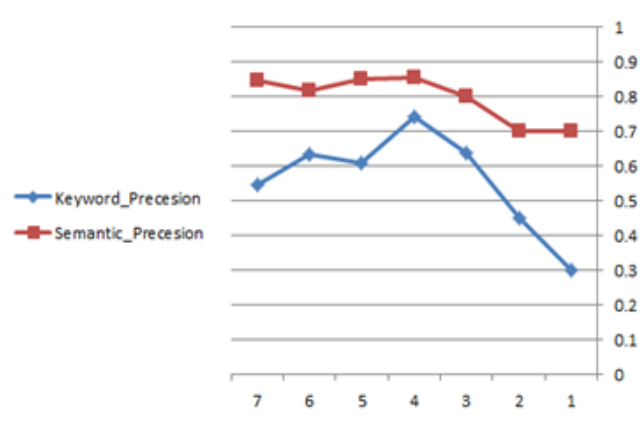

Fig12:Precision difference between keyword and semantic

\section{CONCLUSION}

In this paper,the functioning prototype is presented for semantic and shareable basedrepository of learning objects. Such proposed system allows the retrieval of, not only a perfect LO match, but also of related LO.

To support the proposed approach, we described why current metadata schemes do not cope with current usage of educational material in general, and of learning objects in particular. We described our basis to deploy the repository, which is linked to a coupled system integrating an IS and a LMS.

The proposed approach enables e-learning systems to easily reuse and share semantic learning objectspublished by various systems. It can easy process learning object and can infer a learner should study and what learning objects a system should look. The system can retrieve all the LO, which are relevant while retrieving as few non-relevant $\mathrm{LO}$ as possible.

\section{REFERENCES}

[1] Alian, M. \&AL-Akhras, M. (2010). AdaLearn: An Adaptive E-Learning Environment. ISWSA'10, June 1416, 978-1-4503-0475, ACM.
[2] Zschocke, T. \& Villagrán de León, J. (2010). Towards an Ontology for the Description of Learning Resources on Disaster Risk Reduction. WSKS 2010, Part I, CCIS 111, pp. 60-74, Springer-Verlag Berlin Heidelberg.

[3] Ghaleb, F., Daoud, S., Hasna, A., Jaam, J., El-Seoud, S., El-Sofany, H. \& Alja 'am, J. (2006). E-Learning Model Based On Semantic Web Technology, International Journal of Computing \&Information Sciences Vol. 4, No. 2, August 2006, On-Line. Pages $63-71$.

[4] Liu, J., Huang, B. \&Chao, M. (2005). The Design of Learning Object Authoring Tool Based on SCORM. Proceedings of the Fifth IEEE International Conference on Advanced Learning Technologies (ICALT'05). 07695-2338-2/05, IEEE.

[5] Wiley, D. A. (2000). Connecting learning objects to instructional design theory: A definition, a metaphor, and a taxonomy. In D. A. Wiley (Ed.), The instructional use of learning objects. Retrieved 2 - 17 - 2005 from: http://reusability.org/read/chapters/wiley.doc.

[6] Lerma, C. (2007). Creating Learning Objects. Proceedings of the 2007 Informing Science and IT Education Joint Conference.

[7] Roy, D. (2006). Automatic Annotation of Learning Materials for E-learning. Ph.D Thesis, Department of Computer Science and Engineering Indian Institute of Technology, Kharagpur.

[8] Vidal, C., Navarrete, A., Menéndez D, V., Gonzalez, A. \&Prieto, M. (2010). Metadata and Ontologies in Learning Resources Design. M.D. Lytras et al. (Eds.): WSKS 2010, Part I, CCIS 111, pp. 105-114, SpringerVerlag Berlin Heidelberg.

[9] Draft Standard for Learning Object Metadata. IEEE P1484.12.1, IEEE Learning Technology Standards Committee(2002),

http://ltsc.ieee.org/wg12/files/LOM_1484_12_1_v1_Fina 1_Draft.pdf.

[10] Mihal, V. \& Bielikova, M.. (2009). AnApproach to Annotation of Learning Texts on Programming within a Web-Based Educational System. 2009 Fourth International Workshop on Semantic Media Adaptation and Personalization. 978-0-7695-3894-5/09, IEEE.

[11] Reeve, L. \&Han. H. (2006). A Comparison of Semantic Annotation Systems for Text-Based Web Documents. In the publication, Web Semantics Ontology edited by David Taniar and Johanna Wenny Rahayu, Idea Group Inc.

[12] Tang, J., Zhang, D., Yao, L. \& Li, Y. (2009), Automatic Semantic Annotation Using Machine Learning. In: The Semantic Web for Knowledge and Data Management. DOI: 10.4018/978-1-60566-028-8.ch006. IGI Global.

[13] Yen, Neil, Shih, T. \&Jin, Q. (2010). A New Paradigm of Ranking \& Searching in Learning Object Repository. MTDL'10, October 29, 2010, ACM 978-1-4503-01589/10/10.

[14] Ding, G. \& Xu, Na. (2010). Automatic semantic annotation of images based on Web data. 2010 Sixth International Conference on Information Assurance and Security. 978-1-4244-7409-7/10, IEEE. 
[15] Jellouli, I. \& El Mohajir, M. (2009). Towards automatic semantic annotation of data rich Web pages. 9781-42442865-6/09, IEEE.

[16] Saini, P., Ronchetti, M. \&Sona. D. (2006). Automatic Generation of Metadata for Learning Objects. Proceedings of the Sixth International Conference on Advanced Learning Technologies (ICALT'06). 0-76952632-2/06, IEEE.

[17] Eric, C., Gagnon, M. \& Ozell, B. (2011). Automatic Semantic Web Annotation of Named Entities. C. Butz and P. Lingras (Eds.): Canadian AI 2011, LNAI 6657, pp. 74-85, 2011. Springer-Verlag Berlin Heidelberg.

[18] Mendenhall, A., Kim, C. \& E. Johnson, T. (2011). Implementation of an Online Social Annotation Tool in a College English Course. D. Ifenthaler et al. (eds.), Multiple Perspectives on Problem Solving and Learning in the Digital Age, DOI 10.1007/978-1-4419-7612-3_20, Springer Science+Business Media.

[19] Sun, S., Frank, Y., Hung, Y, Chang, C., Chen, K., ShihSian, C., Wang, H. \& Liao, H. (2011). Automatic Annotation Of Web Videos. 978-1-61284-350-6/11, IEEE.

[20] Kremen,P., Blaško, M. \&Kouba, Z. (2009). Semantic Annotation of Objects, In: Handbook of Research on Social Dimensions of Semantic Technologies and Web Services. DOI: 10.4018/978-1-60566-650-1.ch011. IGI Global.

[21] Dicheva, D. (2008), Ontologies and Semantic Web for ELearning.In: "Handbook on Information Technologies for Education and Training, International Handbooks on Information Systems, pp 47-65, Springer Berlin Heidelberg.

[22] Różewski, P., Kusztina, E., Tadeusiewicz, R. \& Zaikin, O. (2011). Learning Object Methodology. In: Intelligent Open Learning Systems Concepts, Models and Algorithms. Springer-Verlag Berlin Heidelberg.

[23] Gasevic, D., Jovanovic J., and Devedzic V. (2007). Ontology-Based Annotation of Learning Object Content. Interactive Learning Environments Vol. 15, No. 1, April 2007, pp. 1 - 26. Taylor \& Francis.

[24] Zhang, T. \&Shen, R. (2009). Learning Objects Automatic Semantic Annotation by Learner Relevance Feedback. Biomedical Engineering and Informatics, 2009. BMEI '09. $2^{\text {nd }} .978-1-4244-4134-1 / 09$, IEEE.

[25] Dingli,A. (2011). Introducing Annotation. In: Knowledge Annotation: Making Implicit Knowledge Explicit, ISRL 16, pp. 3-17. Springer-Verlag Berlin Heidelberg.

[26] Kiryakov, A.,Popov, B., Terziev, I., Manov, D.\&Ognyan, D. (2004). Semantic annotation, indexing, and retrieval. Web Semantics: Science, Services and Agents on the World Wide WebVolume 2, Issue 1, 1 December 2004, Pages 49-79. Elseiever.

[27] Devedžic, V. (2006).Semantic Web And Education, ISBN: 0-387-35417-4, Springer Science+Business Media, LLC.

[28] Shafrir, U. \& Etkind, M. (2006).E-Learning for depth in the Semantic Web, British Journal of Educational Technology, Vol 37 No 32006 425-444, Published by
Blackwell Publishing, 9600 Garsington Road, Oxford OX4 2DQ, UK and 350 Main Street, Malden, MA 02148, USA.

[29] Karin, B., Antonio, M.\&Walt, T. (2007), Semantic Web: Concepts, Technologies and Applications, e-ISBN: 9781-84628-710-7, Springer-Verlag London Limited.

[30] Liu, J., Huang, B. \&Chao, M. (2005). The Design of Learning Object Authoring Tool Based on SCORM. Proceedings of the Fifth IEEE International Conference on Advanced Learning Technologies (ICALT'05). 07695-2338-2/05, IEEE.

[31] McGreal, R. (2009). Learning Objects and Metadata Understanding the field. International Workshop on Technology for Education (T4E), Aug 4-6, 2009, Bangalore. 978-1-4244-5505-8/09, IEEE.

[32] Singh, G., Bernard, M. \& Gardler, R. (2004). Creating sharable learning objects From Existing Digital Course Content. WCAE '04 Proceedings of the 2004 workshop on Computer architecture education.ACM.

[33] Morgado, E., Peñalvo, F., Ruiz, Á., Rego, H. \& Moreira, T. (2008). Learning Objects for eLearning Systems. WSKS 2008, CCIS 19, pp. 153-162. Springer-Verlag Berlin Heidelberg.

[34] Mohan, P., Brooks, C. (2003). Learning Objects on the Semantic Web. The 3rd IEEE International Conference on Advanced Learning Technologies (ICALT'03). 07695-1967-9/03,IEEE

[35] Abad, C. (2008). Learning Through Creating Learning Objects Experiences with a Class Project in a Distributed Systems Course, ITiCSE'08, June 30-July 2, 2008, Madrid, Spain, Copyright 2008 ACM 978-1-60558-1156/08/06

[36] Mavrommatis,G. (2008). Learning objects and objectives towards automatic learning construction, European Journal of Operational Research 187 (2008) 1449-1458, doi:10.1016/j.ejor.2006.09.024, Elsevier B.V.

[37] Sharable Content Object Reference Model, http://www.adlnet.gov/Technologies/scorm/default.aspx.

[38] Neven, F. \& Duval, E. (2002), Reusable Learning Objects: a Survey of LOM-Based Repositories, Multimedia'02, December 1-6, 2002, Juan-les-Pins, France, ACM 1-58113-620-X/02/0012.

[39] Kiryakov, A., Popov, B., Terziev, I., Manov, D. \& Ognyanoff, D. (2005).Semantic annotation, indexing, and retrieval. Elsevier's Journal of Web Semantics.

[40] Fouad, K., Nagdy, N. \& Harb, H. (2013). Adaptive ELearning System Based on Semantic Search and Recommendation in the Arab World. In: Albadri, F. Information Systems Applications in the Arab Education Sector. DOI: 10.4018/978-1-4666-1984-5.ch018. IGI Global.

[41] Reeve, L. \& Han, H. (2005). Survey of Semantic Annotation Platforms. SAC'05, March 13-17, 2005 , Santa Fe, New Mexico, USA. ACM 1-58113-964 0/05/0003.

[42] Li, H., Tian, Y., Ye, B. \& Cai, Q. (2010). Comparison of Current Semantic Similarity Methods in WordNet. 20IO International Conference on Computer Application and 
System Modeling (ICCASM 2010). 978-1 -4244-72376/10, IEEE.

[43] Fellbaum, C. (2010). WordNet. Theory and Applications of Ontology: Computer Applications, 231, PP: 231-243, Springer Science+Business Media B.V.

[44] Lee, W. \& Mit, E. (2011). Word Sense Disambiguation By Using Domain Knowledge. International Conference on Semantic Technology and Information Retrieval. 9781-61284-353-7/11, IEEE.

[45] U. Siti, A. Rohiza, M. Shakirah. (2010). Ontology of Programming Resources for Semantic Searching of Programming Related Materials on the Web. 978-14244-6716-7110, IEEE.

[46] H. YUN, J. XU, M. J. XIONG. (2009). Development of Domain Ontology for E-learning Course, 978-1-42443930-0/09, IEEE.
[47] Stavredes, T. (2005). Shareable Learning Objects. In: Encyclopedia of Distance Learning. DOI: 10.4018/9781-59140-555-9.ch244. IGI Global.

[48] The Jorum Team. 2005. Report on Open Source Learning Object Repository Systems. Signed-Off by JISC.

http://www.jorum.ac.uk/squeezy/cms/docs/pdf/JORUM_ osswatch_final.pdf.

[49] LIU, X., ZHOU, Y. \& ZHENG, R. (2008). Measuring Semantic Similarity within Sentences. Proceedings of the Seventh International Conference on Machine Learning and Cybernetics, Kunming, 12-15 July 2008. 978-14244-2096-4/08, IEEE. 